\section{Investigation of anxiety and depression in patients with chronic diseases}

\author{
Evangelia-loanna Gerontoukou,1 \\ Sofia Michaelidoy, 1 Maria Rekleiti,2 \\ Maria Saridi,2 Kyriakos Souliotis 1 \\ 1 Faculty of Social Sciences, University of \\ Peloponnese, Korinthos; ${ }^{2}$ General \\ Hospital of Korinthos, Greece
}

\section{Abstract}

The health of an individual depends on both his/her physical and psychological condition. In recent years it has been observed that chronic patients have frequently an affected psycho-emotional state. The purpose of this study is to investigate anxiety and depression in patients with chronic diseases and the correlation of the results with daily physical activity levels and individual health levels, as well comorbidity. This study included patients with chronic diseases that were treated in a local general hospital or were visiting often outpatient clinics of the same hospital due to their condition. The sample in this particular study included 204 patients; 118 of them were women and 86 men. From the total sample that participated in our research, 118 (57.8\%) were females and the majority of the participants were secondary/basic education graduates (67\%), married (71\%), living in urban areas (53\%). Hypertension was the most frequent chronic disease in our sample, followed by hypercholesterolemia and diabetes mellitus. Comparing the occurrence of depression and anxiety symptoms in both questionnaires in relation to the expected frequency in the general population, significant levels of depression and anxiety symptoms were recorded. Taking into consideration the findings of this research, anxiety and depression symptoms can have profound effects regarding the control of chronic diseases, the patients' quality of life and their general health.

\section{Introduction}

In recent years there have been several studies about the investigation of anxiety in patients with chronic diseases, it has been observed that chronic patients present frequently some disruption of their psycho-emotional state. The review of Greek and international literature on databases like PubMed, Scopus, IATROTEK shows the strong correlation between anxiety, depression and chronic disease. In point of fact a recent Greek study on anxiety and depression in patients with coronary artery disease, found that $25.5 \%$ of the subjects experienced moderate stress levels and $32.7 \%$ high stress levels, while $19.1 \%$ experienced moderate depression, and $20 \%$ severe depresion. ${ }^{1}$

Investigation concerning health perceptions of patients with chronic kidney disease who are undergoing hemodialysis or peritoneal dialysis, showed that patients undergoing hemodialysis had a higher rating for answers about their personal control over their health, while changes in the socio-demographic variables were correlated with high levels of depression regarding controlling their situation. ${ }^{2}$ The existence of increased rates of anxiety and depression was also confirmed by another Greek qualitative study regarding renal patients. ${ }^{3}$

In a study which used Zung's scale for the investigation of depression signs in patients with diabetes in relation to its complications, $50 \%$ of the diabetic patients showed mild depression symptoms and 20\% moderate symptoms, while female gender and older age had had a statistically significant correlation with depression. 4

A study carried out in patients with chronic diseases in order to treat their anxiety and help them accept their disease, showed that patients with a neurological disease frequently fail to manage their anger compared to those who suffer from other chronic diseases. Women control their anger more easily than men, while men perform better at managing their anxiety. Unmarried persons can control their emotions and anger at a greater rate than married persons. It is worth noting that the levels of emotional self-control are associated with sex, age and education level. 5

A study undertaken to investigate the incidence of mental disorders in patients monitored in Pain Clinics and chronic orthopedic disorders showed that chronic diseases were significantly associated with the occurrence of psychiatric diseases. $57.1 \%$ of patients hospitalized in pain clinics showed more depression episodes than patients hospitalized in chronic orthopedic hospitals (20.2\%). Among the four subclasses of anxiety, phobia was common to both patient groups (23.2\% vs $5.9 \%$ respectively). Patients in both cases appear to exhibit depression symptoms at higher rates and less stress symptoms. ${ }^{6}$

The investigation of stress and depression levels in patients with chronic obstructive lung disease and heart problems is also important. The results of a systematic review (1994-2009) show that up to $80 \%$ of patients with chronic obstructive lung disease will develop depression symptoms, and $74 \%$ of them may develop stress symptoms as well. In comparison patients with heart problems show up to $60 \%$
Correspondence: Maria Rekleiti, General Hospital of Korinthos, 27 Nikomideias str, GR20100, Korinthos, Greece.

Tel.: +30.274.136.1910

E-mail: mrekliti@gmail.com

Key words: Anxiety; chronic diseases; depression; hypertension.

Contributions: the authors contributed equally.

Conflict of interest: the authors declare no potential conflict of interest.

Conflict of interests: the authors report no conflict of interests.

Received for publication: 21 November 2013. Revision received: 30 January 2014.

Accepted for publication: 30 January 2014.

This work is licensed under a Creative Commons Attribution NonCommercial 3.0 License (CC BYNC 3.0).

(C) Copyright E-I. Gerontoukou et al., 2015 Licensee PAGEPress, Italy

Health Psychology Research 2015; 3:2123 doi:10.4081/hpr.2015.2123

depression and $45 \%$ anxiety. Both of these conditions are correlated to higher mortality rates and lower quality of life. Specialists report that patients with chronic diseases (either lung or heart problems) and depression symptoms are 2.5 times more likely to have impaired mobility, in comparison with depression-free chronic patients. ${ }^{7}$

According to a study regarding the effects of stress and depression in patients with breathing disorders, $65 \%$ of the patients seem to have anxiety and depression problems, a fact that is associated statistically with breathing disorders. ${ }^{8}$

An American study that investigated depression and despair feelings with the possibility of ischemic heart disease in a group of adults, has found that $11.1 \%$ of them had shown signs of depression, $10.8 \%$ of them mild despair feelings and $2.9 \%$ of them had serious despair feelings. Depression and despair was more common in women, non-Caucasian people, singles, smokers and people with low education levels. Depression signs are connected with a possibility of death from ischemic heart problems (relative risk: 1.5) but the risk of ischemic heart problems to people with low despair (relative risk: 1.6) and serious despair (relative risk: 2.1) is even higher. ${ }^{9}$

A study in China regarding depression in patients with chronic diseases has shown that $34.7 \%$ of the participants had depression and that women were more prone to depression while males had an easily changing mood. 10 
A study that took place in Pakistan regarding the effect of psychosocial factors as risk factors for ischemic heart problems, has shown a significant correlation among psychosocial factors and the occurrence of ischemic heart problems. Anger was the most important risk factor, although women aged between 35-55 years were protected against heart conditions because of their hormones, something in agreement with similar findings from other studies. ${ }^{11}$ From the studies mentioned, it becomes clear that chronic diseases affect the psychology of the patient. A wide epidemiological study that will include most of the chronic diseases, using the same instruments that detect anxiety and depression, in order to yield results regarding chronic patients, is necessary. Also the assessment of the patients' needs and the systematic management of anxiety and depression symptoms must be a vital part of the treatment as this will lead to a full bio-psychosocial approach and thus a positive course regarding the disease. For this reason this project has been designed. The diagnosis of anxiety and depression in people those are sick with physical diseases is a big challenge for healthcare professionals. And that happens because the main symptoms of these particular psychological disorders (apathy, lack of concentration, interest, a feeling of tiredness all the time, sleeping and eating disorders) is possibly connected with their physical disease, which consequently affects their overall quality of life and their compliance to medical instructions and treatment. The aim of the present study was to record comorbidity in chronic patients, assess their anxiety and depression levels, as well as compare the results with demographical and other data.

\section{Materials and Methods}

The study included chronic patients under treatment in a local general hospital or were visiting often outpatient clinics of the same hospital regarding their condition. The sample included 204 patients: 118 of them were women and 86 men with chronic diseases. The sample was collected by random sampling. The authors included those patients that were visiting the outpatient clinics on Tuesdays and Thursdays only. The collection of the data begun on March 2013 and was completed by June 2013. The data were collected by using anonymous questionnaires (self reference). Overall, 220 questionnaires were handed out, and 204 of them were returned fully completed (response rate $92.72 \%$ ).

The research protocol was granted approval by the hospital review board. The chief doctors and the head nurses were informed about the study, so that the clinics would operate normal- ly, considering the special needs of the participants.

\section{Exclusions from the study}

The following persons were excluded from the study: i) persons that were working or were being trained in the same hospital even if they attended the outpatient clinics for their own medical problems; ii) persons that did not sign the consent form and consequently did not want to take part in the study; iii) persons that during the interviews or the completion of the questionnaire opted out of the study for any reasons whatsoever; iv) persons whose mother-tongue was other than Greek; v) persons with mental disorders.

\section{Questionnaire}

The main tool of the collection of the data was a self-reference questionnaire that included 3 parts.

First part: a 9-item structured questionnaire regarding the patients' general profile and demographics was used under the authors' authorization. 4

Second part: The Zung self-rating depression scale was used for the assessment of depression signs. ${ }^{12}$ The Greek version of the ZDRS was used due to its high sensitivity, specificity and repeatability. The ZDRS was used as a screening tool and not as a counseling session substitute. ${ }^{13}$ It includes 20 items that cover emotional, psychological and physical symptoms. The patient states how often s/he experiences one particular symptom (not at all, sometimes, often, and always). The answers correspond to a specific score (not at all: 1, sometimes: 2 , often: 3 , always: 4 ) and the total score helps to assess the depression symptoms. The severeness of depression according to the Zung scale (SDS) is then calculated and used in the statistical analysis. High SDS scores indicate severe depression $(86 \leq S D S)$, while low scores $(\mathrm{SDS}<55)$ are a sign of a normal emotional state. ${ }^{14,15}$

Third part: The Greek version of the Delusions-Symptoms-States Inventory State of Anxiety and Depression scale (DSSI/sAD) by Bedford and Fould, was used for the assessment of anxiety and depression symptoms. ${ }^{16-18}$ The instrument included 14 questions, seven of them regarding stress and 7 regarding depression. It is either used as a control test for detecting psychiatric symptoms or as an evaluation tool for anxiety and depression symptoms. Every question includes two parts. The first part evaluates the mere existence of a symptom and the second part assesses to what extent that particular symptom affects the patient. The combination of those two parts leads to a score, where $0=$ the symptom does not exist, $1=$ the symptom exists creating a minor discomfort, $2=$ the symptom exists, creates a moderate discomfort, $3=$ the symptom exists, creating major discomfort. By adding the two (anxiety and depression) subscales' scores the overall scores can be calculated.

\section{Ethics}

The names of the participants were not published in any way not only during the time that the study was taking place but also during the analysis and when the results were announced. The participation was voluntary and the operation of the hospital was not disrupted at any case. In addition, there wasn't any financial burden to the hospital or the participants. The findings of the study will be used only for scientific reasons and they should shed some light on dealing with chronic patients and managing their anxiety and depression.

\section{Statistical analysis}

A descriptive and inferential analysis took place. For the comparison of categorical data, Pearson's $\chi^{2}$, and Yates' correction $\left(\mathrm{X}_{c}{ }^{2}\right)$ were employed; whenever even one result was $<1$, or the $\chi^{2}$ test simply could not be used, Fisher's Exact Test, the Likelihood Ratio or the Linear by linear association were used. Statistical significance was set to $\mathrm{P}<0.05$. The SPSS 17.0 software was used for the statistical analysis.

\section{Results}

Among the participants, 118 were female (57.8\%). The majority of the participants were high school graduates (67\%), married (71\%), living in urban areas (53\%), as shown in Table 1. The average age of the participants was $56.72 \pm 21.09$ years.

Hypertension was the most common chronic condition (36.3\%) in our sample, followed by hypercholesterolemia and diabetes mellitus, as shown in Table 2. According to the questionnaire by Bedford \& Foulds, the majority of the participants (54\%) appear to have possible depression, and also some of their symptoms point towards anxiety disorder, as shown in Figure 1A,B. According to the Zung's questionnaire, the majority of the participants $(65 \%)$ seem to have marginal or possible depression (Figure 2). Comparing the frequency of depression symptoms in both questionnaires it was found that the distribution was comparable without any statistically significant difference. Comparing the frequency of depression and anxiety symptoms in both questionnaires in relation to the expected frequency in the general population, higher rates of depression and anxiety symptoms were found, as shown in Figure $2(\mathrm{P}<0.001)$. Comparing the emergence of depression symptoms between the two genders, it was fund that females develop 
more symptoms compatible with depression (47.5\% vs $31.7 \%$ ) compared to males, although this difference failed to reach the level of statistical significance $\left(\chi^{2}=3.715, \mathrm{P}=0.156\right)$. On the contrary, regarding age, participants 57 years and older have higher rates of depression compared to those younger than 57 years ( $48 \%$ vs $33 \%$ ), a variation marginally statistically significant $\left(\chi^{2}=5.459, \quad \mathrm{P}=0.065\right)$. Moreover, participants 57 years and older, have higher anxiety rates than those younger than 57 years (68\% vs 49\%), a statistically significant variation ( $\mathrm{LR}=4.619, \mathrm{P}=0.097$ ).

The existence of a large number of children seemed to have a positive correlation with anxiety, and the likelihood of anxiety disorder increases in parallel with the number of children.

A marginally significant correlation between anxiety and education level was found, with primary education level participants having a $86 \%$ likelihood of manifesting anxiety disorder, while for higher education graduates the rates are close to zero and for participants of intermediate education levels the rates are under $68 \%$. However, when education levels are split in just two broad categories (highest/higher and primary/secondary), no statistically significant difference can be observed, although persons of primary/secondary education level seem to have a higher likelihood of developing an anxiety disorder.

Unmarried persons appeared to have an increased likelihood of depression, as most of them (94\%) seemed to have marginal symptoms and possible depression, while for the participants who had another marital status (married/other), the respective rate did not exceed $67 \%$.

Regarding chronic conditions, participants who suffered from coronary artery disease had had higher anxiety rates (71.4\% anxiety disorder) compared with the rest of the participants (53.4\% anxiety disorder). The variation was found to be statistically significant: $L R=6.733$, $\mathrm{P}=0.035$.

As shown in Table 3 , there was a satisfactory correlation among the two scales, and the distribution of the respondents in all three categories (normal feeling, marginal symptoms and possible depression) was similar, although in this study it seemed that Zung's scale tended to overrate marginal symptoms.

Finally, there was a significant difference between smokers and non-smokers. $49 \%$ of non-smokers experienced a normal emotional status, compared to $31 \%$ of those who used to smoke. Nevertheless, there was no statistically significant difference regarding anxiety levels.

\section{Discussion}

In the present study, the majority of the participants were female with average age of $56.72 \pm 21.09$ years, basic and secondary education level, and married, who lived in an urban area. Regarding chronic diseases, a third of the sample had had hypertension followed by diabetes and hypercholesterolemia. In the course of the study it was found that the majority of the participants developed depression symptoms and signs of anxiety disorders. Females had had higher depression rates; similarly, participants older than 57 years of age had had higher depression and anxiety rates.

In most studies concerned with anxiety and depression in chronic patients, female participants routinely score higher depression/anxiety rates, probably because women tend to respond more easily in self-reference studies. In the present study, females also had had higher depression rates, a finding which is in agreement with other relevant Greek and international studies. 4,10

Leung et al. by using a sample of chronic patients whose most common medical condition was hypertension, found that almost $35 \%$ of their sample developed depression symptoms, a finding pretty similar to ours. ${ }^{4}$ Another study in patients with coronary disease found that $58.2 \%$ of the participants had had medium and high levels of anxiety, a finding that is almost the same with the respective anxiety levels in our study, which is $56 \%$. $^{1}$ Several studies have shown the emergence of anxiety in patients with coronary diseases, mainly in those that had suffered an acute coronary episode. ${ }^{19}$ Furthermore, patients with both coronary disease and depression, seemed to run a higher risk of a new cardiac incident. ${ }^{20}$

Yohannes et al. in a systematic review of studies published between 1994 and 2009, found that patient with chronic diseases, and especially patients with obstructive lung disease and cardiovascular diseases can have particularly high rates of anxiety and depression, thus increasing mortality rates and decreasing the quality of life levels. ${ }^{7}$ Another study by Janowski et al. with a sample of 300 chronic patients found inadequate anxiety control by correlating sex and age. It was also found that women can control easier their feelings than

Table 3. Correlation of scales Bedford \& Fould and Zoung for depression.

\begin{tabular}{|c|c|c|c|c|}
\hline \multirow{2}{*}{$\begin{array}{l}\text { Depression scale } \\
\text { (Bedford \& Fould) }\end{array}$} & \multicolumn{3}{|c|}{ Depression scale (Zoung) } & \multirow[t]{2}{*}{ Total } \\
\hline & $\begin{array}{l}\text { Normal } \\
\text { feeling }\end{array}$ & $\begin{array}{l}\text { Borderline } \\
\text { symptomatolc }\end{array}$ & $\begin{array}{l}\text { Mild } \\
\text { pression }\end{array}$ & \\
\hline Normal feeling & 30 & 34 & 28 & 92 \\
\hline Borderline symptomatology & 10 & 12 & 6 & 28 \\
\hline Possible depression & 32 & 32 & 20 & 84 \\
\hline Total & 72 & 78 & 54 & 204 \\
\hline
\end{tabular}

Likelihood ratio: $0.673, \mathrm{P}=0.955$.

men. The existence of high anxiety levels in that study is the same with the levels found in our study, as $56 \%$ of the participants showed signs of a possible anxiety disorder. ${ }^{5}$ Similarly, Wong et al. found that chronic diseases are linked with the emergence of psychiatric conditions. In their study, the whole sample showed high rates of depression and anxiety symptoms, which is in agreement with our findings as well. ${ }^{6}$ In another study by Rekleiti et al. with a sample of chronic diabetic patients, $50 \%$ of them had developed a mild level of depression symptoms, and $20 \%$ of them had developed medium level depression symptoms, whereas female gender, older age and

Table 1. Demographic characteristics of the sample.

\begin{tabular}{lcc} 
Variable & N & $\%$ \\
Sex & & \\
$\quad$ Man & 86 & 42.2 \\
$\quad$ Women & 118 & 57.8 \\
Educational level & & \\
$\quad$ Highest & 46 & 22.6 \\
Higher & 20 & 10.0 \\
Secondary & 48 & 24.0 \\
$\quad$ Primary & 86 & 43.0 \\
\hline Marital status & & \\
$\quad$ Single & 34 & 16.7 \\
$\quad$ Married & 146 & 71.6 \\
$\quad$ Other & 24 & 11.8 \\
Abode & & \\
$\quad$ Urban areas & 108 & 52.9 \\
Suburban & 54 & 26.5 \\
Rural & 42 & 20.6 \\
\hline
\end{tabular}

Table 2. Incidence of diseases in the sample of the study.

\begin{tabular}{lcc} 
Disease & $\mathrm{N}$ & $\%$ \\
Hypertension & 74 & 36.3 \\
Neurological diseases & 8 & 3.9 \\
\hline Hypercholesterolemia & 42 & 20.6 \\
Coronary artery disease & 28 & 13.7 \\
\hline Diabetes mellitus & 42 & 20.6 \\
Chronic obstructive & 14 & 6.9 \\
pulmonary disease & & \\
\hline Ischemic stroke & 34 & 16.7
\end{tabular}


diabetes-related complications were found to have a significant correlation with depression. 4

An older Norwegian study that had included 291 diabetic patients, had showed that as the chronic diabetic symptoms pile up, so do the symptoms of anxiety and depression.21 Many others studies have also acknowledged the emergence of anxiety and depression symptoms in chronic patients. ${ }^{8-11,17}$

In contrast to our findings, several studies have correlated the emergence of depression and anxiety to family status and number of children. More specifically, married participants with two or more children, because of their responsibilities combined with their special needs for care, usually fail to manage and control their feelings, something that may lead to the emergence of anxiety and depression symptoms. ${ }^{5,22}$ It is noteworthy that in some chronic patients' groups, such as patients with ischemic heart disease and a previous heart attack, the emergence of depression is common among unmarried patients, perhaps due to their fear of a new episode in combination with the lack of a family environment, a finding in agreement with ours. ${ }^{9}$ Also, changes in several socio-demographic variables are frequently associated with the increased percentage anxiety and depression rates within chronic patients. ${ }^{2,3}$ As far as age was concerned, according to the relevant literature, patients older than 55 years tend to have higher anxiety and depression rates. $4,8-11 \mathrm{~A}$ substantial finding in our study was the fact that smokers seem to have higher depression rates than non-smokers. This finding is quite common in the international literature regarding not only chronic patients but the general population as well; nevertheless, it is not yet quite clear if an underlying depression leads someone to smoke or vice versa. ${ }^{22,23}$ Many of the studies available are retrospective and have used patient data from several medical facilities. On the other hand, the present study was a prospective one with an a priori well-defined research protocol. All data was carefully collected by the research team, so that only complete, high-quality data were collected. The relatively small size of the sample, mainly because of the limited time available for the completion of this specific study, constitutes the main limitation in our study. Our findings could potentially be generalized, mainly because they are in agreement with the international literature, nevertheless it remains doubtful whether they describe accurately the general population of chronic patients. The research team aims at designing and launching a pure epidemiological study, which will investigate a representative sample of a larger area with the appropriate stratification of demographics of the sample, in order for the findings to reach higher generalizability and validity.
A

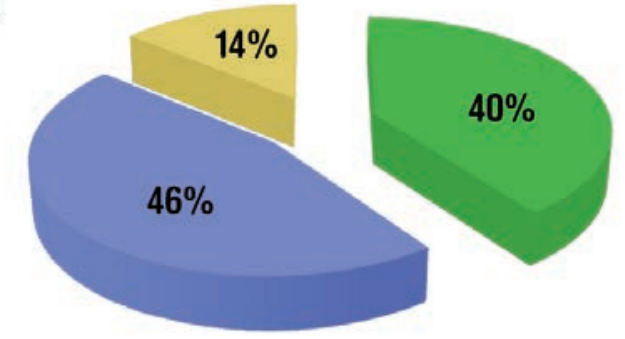

- Possible depression

n Normal feeling

Marginally depression

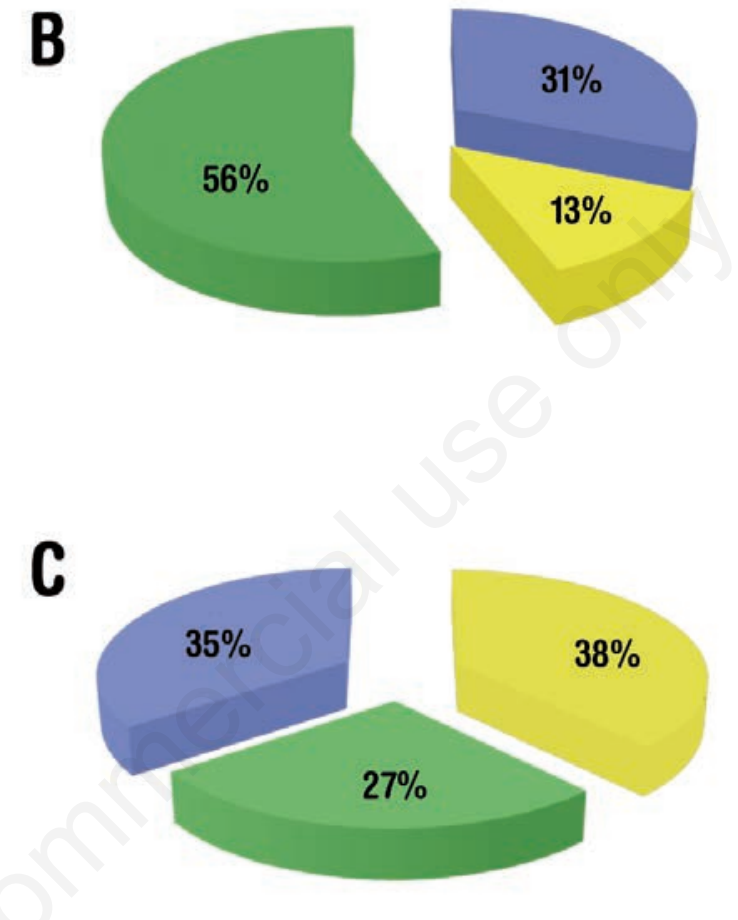

- Normal feeling

- Marginally symptomatology

- Possible anxiety disorder
Marginally
symptomatology

= Possible depression

- Normal feeling

Figure 1. Categorization of responses to the questionnaire Bedford \& Fould (A, scale depression; B, anxiety scale) and the questionnaire Zoung (C).

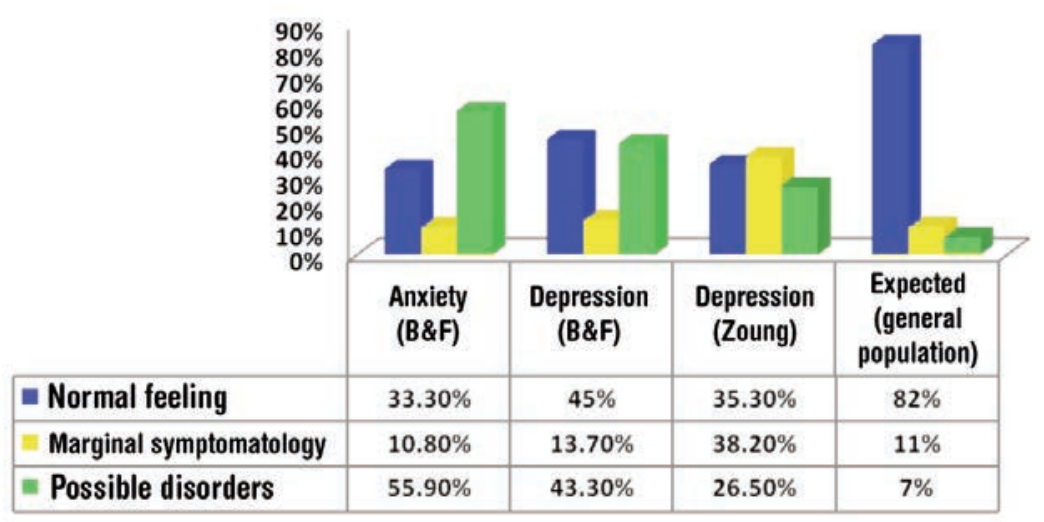

Figure 2. Comparative presentation of the frequency of symptoms of anxiety and depression in both questionnaires compared with the expected frequency in the general population. 


\section{Conclusions}

To sum up, despite the small sample of our patients, the present study demonstrated that there is a relation between chronic diseases and the emergence of depression and anxiety symptoms. Our findings are for the most part in agreement with the relevant international literature. Taking into consideration the findings of the present study, we suggest that anxiety and depression symptoms may affect the patients' ability to control their condition, their quality of life and their overall health levels.

The investigation of those factors that could trigger anxiety and depression symptoms in chronic patients, by respecting their personality and identifying other underlying causal factors, as well as the forming a protective framework for those patients, are crucial factors in order for them to adjust their lifestyle according to the limitations imposed by their disease, and also maintain good quality of life.

Modern societies due to the advancement of science and the increasingly aging populations are expected to have higher chronic patient rates. Thus, each country's health system should monitor closely high-risk persons and intervene in the risk factors as early as possible in order to reduce symptoms and complications such as psycho-emotional disorders, anxiety and depression, and ultimately provide improved levels of health care.

\section{References}

1. Polukandrioti M, Gouvenos I, Michalis L, et al. The effects of anxiety and depression on the needs of patients hospitalized with coronary disease. Arch Hell Med 2012;29:33-43.

2. Theophilou P. Quality of life and mental health in hemodialysis and peritoneal dial- ysis patients: the role of health beliefs. Int Urol Nephrol 2012;44:245-53.

3. Minos C, Rekleiti M, Saridi M, et al. Factors affecting quality of life in endstage renal disease patients on hemodialusis. Hell J Nurs Sci 2012;5:64-71.

4. Rekleiti M, Roupa Z, Kyriazis I, et al. Selfassessment of depression in patients with diabetes mellitus and its correlation with complications. Arch Hell Med 2012;29:599605.

5. Janowski K, Kurpas D, Kusz J, et al. Emotional control, styles of coping with stress and acceptance of illness among patients suffering from chronic somatic diseases. PLoS One 2013;8:e63920.

6. Wong WS, Chen PP, Yap J, et al. Chronic pain and psychiatric morbidity: a comparison between patients attending specialist orthopedics clinic and multidisciplinary pain clinic. Pain Med 2011;12:246-59.

7. Yohannes AM, Willgoss TG, Baldwin RC, Connolly MJ. Depression and anxiety in chronic heart failure and chronic obstructive pulmonary disease: prevalence, relevance, clinical implications and management principles. Int $\mathrm{J}$ Geriatr Psychiatry 2010;25:1209-21.

8. Kunik ME, Roundy K, Veazey C, et al. Surprisingly high prevalence of anxiety and depression in chronic breathing disorders. Chest J 2005;127:1205-11.

9. Anda R, Williamson D, Jones D, et al. Depressed affect, hopelessness, and the risk of ischemic heart disease in a cohort of US adults. Epidemiology 1993;4:285-94.

10. Leung K, Lue B, Lee M, Tang LY. Screening of depression in patients with chronic medical diseases in a primary care setting. Fam Pract 1998;15:67-75.

11. Rafique R, Amjab N. Psychosocial factors as predictors of early onset ischemic heart disease in a sample of Pakistani women International. J Res Stud Psychol 2012;1:17-27.

12. Zung WWK. A self-rating depression scale.
Arch Gen Psychiatry 1965;12:63-70.

13. Fountoulakis KN, lacovides A, Samolis S, et al. Reliability, validity and psychometric properties of the Greek translation of the Zung depression rating scale. BMC Psychiatry 2001;1:6.

14. Zung WWK. A cross-cultural survey of symptoms in depression. Am J Psychiatry 1969;126:116-21.

15. Zung WWK. A cross-cultural survey of depressive symptomatology in normal adults. J Cross-Cult Psychol 1972;3:177-83.

16. Bedford A, Foulds GA, Sheffield BF. A new personal disturbance scale: DSSI/sAD. Br J Soc Clin Psychol 1976;15:387-94.

17. Krommydas GC, Gourgoulianis KI, Angelopoulos NV, et al. Depression and pulmonary function in outpatients with asthma. Respir Med 2004;98:220-4.

18. Roupa Z, Tatsiou I, Tsiklitara A, et al. Anxiety and depression among elderly in the community. Intersci Health Care 2009;1:61-6.

19. Babatsikou F, Zanitsanou A. Epidemiology of hypertension in the elderly. Health Sci 2010;4:24-30.

20. Lykouras L, Alevizos B, Michalopoulou P, Rabavilas A. Obsessive-compulsive symptoms induced by atypical antipsychotics: a review of the reported cases. Prog Neuropsychopharmacol Biol Psychiatry 2003;27:333-46.

21. Enqum A. The role of depression and anxiety in onset of diabetes in a large population-based study. J Psychosom Res 2007;62:31-8.

22. Kyloudis P, Rekliti M, Kyriazis I, et al. Probing stress and depression in mental health professionals at the general hospital in Greece. Eur Psychiatry 2010;25:731.

23. Michal M, Wiltink J, Reiner I, et al. Association of mental distress with smoking status in the community: results from the Gutenberg health study. J Affect Disord 2013;146:355-60. 